\title{
Surface-Controlled Metal Oxide Resistive Memory
}

\author{
Jr-Jian Ke, Kyoko Namura, José R. D. Retamal, Chih-Hsiang Ho, Haruhiko Minamitake, Tzu-Chiao \\ Wei, Dung-Sheng Tsai, Chun-Ho Lin, Motofumi Suzuki \& Jr-Hau He*
}

\begin{abstract}
To explore the surface effect on resistive random-access memory (ReRAM), the impact of surface roughness on the characteristics of $\mathrm{ZnO}$ ReRAM were studied. The thickness-independent resistance and the higher switching probability of $\mathrm{ZnO}$ ReRAM with rough surfaces indicate the importance of surface oxygen chemisorption on the switching process. Furthermore, the improvements in switching probability, switching voltage and resistance distribution observed for ReRAM with rough surfaces can be attributed to the stable oxygen adatoms under various ambience conditions. The findings validate the surface-controlled stability and uniformity of ReRAM and can serve as the guideline for developing practical device applications.
\end{abstract}

Index Terms - ZnO, oxygen chemisorption, surface roughness, resistive random-access memory.

\section{INTRODUCTION}

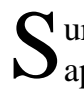
urface effect is a double-edged sword for the device application of metal oxide. Surface effects resulting from the gas molecules chemisorption-induced surface band bending significantly influence electronic and optoelectronic properties of metal oxide devices [1]. For example, benefited from the surface band bending of the $\mathrm{ZnO}$ due to the chemisorbed $\mathrm{O}_{2}$ molecules, which is around $1.53 \mathrm{eV}$, nanostructured gas sensors and photodetectors have shown remarkable device sensitivity [1]. On the other hand, this surface effect can be detrimental to some device applications, such as oxide thin film transistors because of surface effect-induced electrical instability [2].

Recently, metal oxide-based resistive random-access memory (ReRAM) for the non-volatile memory has captured great attention due to the excellent reversible resistive switching. Among the metal oxides currently had being explored for the development of ReRAM, $\mathrm{ZnO}$ has been demonstrated to be a potential candidate. $\mathrm{ZnO}$ is an n-type wide bandgap semiconductor and is highly transparent in the visible spectral region. Its conductivity can be modulated by suitable impurity

This work was supported by the Chung-Hua Institution for Economic Research.

J.-J. Ke, J. R. D. Retamal, T.-C. Wei, C.-H. Lin and J.-H. He are with Computer, Electrical and Mathematical Sciences and Engineering (CEMSE) Division, King Abdullah University of Science \& Technology (KAUST), Thuwal 23955-6900, Saudi Arabia (e-mail: jrhau.he@kaust.edu.sa).

J.-J. Ke and D.-S. Tsai are with the Department of Electrical Engineering, and Graduate Institute of Photonics and Optoelectronics, National Taiwan University, Taipei 10617, Taiwan

K. Namura and M. Suzuki are with Department of Micro Engineering, Kyoto University, Kyoto 606-8501, Japan

C.-H. Ho is with Electrical and Computer Engineering, Purdue University, West Lafayette, IN 47907, USA

Digital Object Identifier xx.xxxx/xxxx.xxxx.xxxxx doping. Therefore it is possible to develop a fully transparent ReRAM based on $\mathrm{ZnO}$. The switching mechanisms of $\mathrm{ZnO}$ ReRAM are mainly attributed to the formation/rupture of defect-based conductive nanofilaments near the electrode/metal oxide interface, which is greatly influenced by the chemisorbed $\mathrm{O}_{2}$ molecules at the surfaces [3]. A great effort has been made to eliminate the detrimental surface effect on the resistive memory switching. It has been reported that by introducing transparent graphene electrodes as a passivation layer, the detrimental surface effect can be eliminated [4]. He et al. had reported that surface modification of $\mathrm{ZnO}$ resistive memory via fluorine and nitrogen doping to replace the oxygen sites leads to superior performance uniformity [5]. However, these optimization methodologies come with additional cost and complexity of fabrication process. Hence, a simple, scalable and cost-effective fabrication method for eliminating the surface effect is needed for practical application of resistive oxide memory.

In this work, the influences of surface roughness on the electrical property of $\mathrm{ZnO}$ ReRAM were carefully investigated. Other than the chemical modification, the physical method of glancing angle deposition (GLAD) was employed to modify the surface roughness of $\mathrm{ZnO}$. It demonstrates that the $\mathrm{ZnO}$-based ReRAM with rough surface possess higher resistive switching probability and are more stable in SET/RESET switching voltage and HRS resistance for various ambience conditions. Furthermore, from the measurement results of field effect transistors (FETs), smaller variation of threshold voltage $\left(\mathrm{V}_{\mathrm{th}}\right)$ is observed for $\mathrm{ZnO}$ with rough surface under various ambience conditions. It shows that the change in oxygen adatoms $\left(\mathrm{O}_{2(\mathrm{ad})}^{-}\right)$ concentration is insignificant at the rough surface which, in turn, leads to a stable electrical property of $\mathrm{ZnO}$. These observations advance the understanding of surface effects and provide valuable insights for future optimization of ReRAM devices.

\section{EXPERIMENT}

100-nm-thick $\mathrm{ZnO}$ thin films were deposited on the $\mathrm{Pt} / \mathrm{Cr} / \mathrm{SiO}_{2} / \mathrm{Si}$ substrates at room temperature (RT) by $\mathrm{rf}$ magnetron sputtering with an $\mathrm{O}_{2}$ working pressure of $6 \times 10^{-1} \mathrm{~Pa}$ and power of $100 \mathrm{~W}$ using a $\mathrm{Zn}$ target. The GLAD with an angle of $70^{\circ}$ and 20 min deposition was employed to modify the surface roughness of $\mathrm{ZnO}$. Surface modification by the GLAD had been reported in the literatures [6]. During the GLAD, the deposition power and pressure are $100 \mathrm{~W}$ and $6 \times 10^{-1} \mathrm{~Pa}$, respectively. In order to measure the electrical properties of the $\mathrm{ZnO}$ films, the top electrodes $\mathrm{Pt}$ with thickness of $100 \mathrm{~nm}$ were deposited on the $\mathrm{ZnO}$ using DC sputtering. To investigate the 
ambient influences on the electrical property, 25 cells were evaluated at RT under air or vacuum condition (10 $0^{-5}$ Torr). Morphology and roughness of the $\mathrm{ZnO}$ surface were investigated by atomic force microscopy (AFM). The back-gate FET configuration measurements $\left(\mathrm{Pt} / \mathrm{ZnO} / \mathrm{SiO}_{2} / \mathrm{n}^{+}-\mathrm{Si}\right)$ were also prepared to study the oxygen chemisorption on the $\mathrm{ZnO}$ surface.

\section{RESULTS AND DISCUSSION}

To distinguish interface and bulk effects, the thickness-dependent electrical properties of initial state (IS), HRS and LRS were investigated. As shown in Fig. 1(a), the resistance of IS increases with the thickness of $\mathrm{ZnO}$ films. The IS of ZnO ReRAM behaves as conventional ZnO-based devices, which consists of $\mathrm{ZnO}$ thin films and electrode/ $\mathrm{ZnO}$ interface connected in series [7]. Hence, the total resistance of $\mathrm{ZnO}$ ReRAM can be obtained by the sum of the resistances of bulk $\mathrm{ZnO}$ and electrode $\mathrm{Pt} / \mathrm{ZnO}$ interface. On the other hand, the resistances of high resistance state (HRS) and low resistance state (LRS) are thickness-independent. The ReRAM switching property relies on the formation/rupture of the conductive nanofilament built by the $\mathrm{V}_{\mathrm{O}}[4,8,9]$. As the $\mathrm{ZnO}$ ReRAM devices were electroformed, the metallic conductive nanofilament dominates the electrical transport within the $\mathrm{ZnO}$ bulk, leading to the thickness independence on HRS and LRS resistance, which indicates that the switching process relies on the resistance changes near the $\mathrm{Pt} / \mathrm{ZnO}$ interface [7], as shown in Fig. 1(b). It shows that the interface plays an important role in the resistive switching functionality.

To study the influences of $\mathrm{Pt} / \mathrm{ZnO}$ interface on the $\mathrm{ZnO}$ ReRAM characteristics, the switching probability was measured under various ambience and surface roughness conditions. Fig. 2 illustrates schematic and corresponding AFM image of $\mathrm{ZnO}$ with flat and rough surfaces. For the $\mathrm{ZnO}$ with flat surface, the root-mean-square (RMS) and max roughness are 2.23 and 16.5, respectively. On the other hand, RMS and max roughness for the rough $\mathrm{ZnO}$ surface are 4.98 and 32.3, indicating a rough surface. As shown in Fig. 3(a), ZnO ReRAM
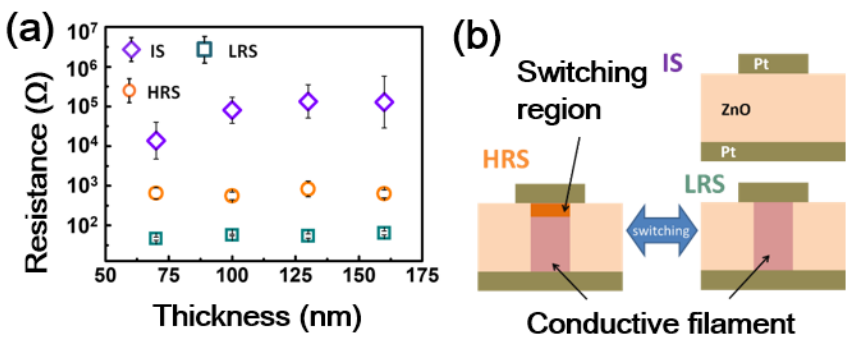

Fig. 1 (a) Thickness dependence of initial state (IS), HRS and LRS. (b) The resistive switching process of $\mathrm{ZnO}$ resistive memory devices.
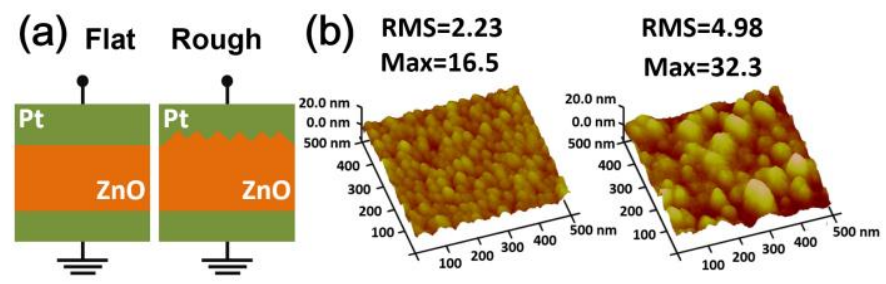

Fig. 2. (a) Schematics of device structure and (b) AFM images of flat and rough surface condition.
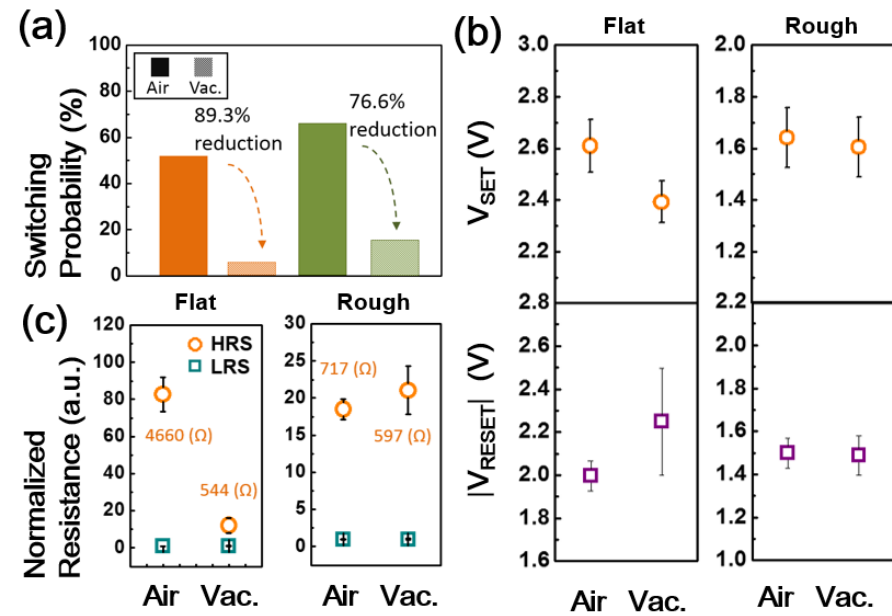

Fig. 3. (a) Resistive switching probability, (b) SET/RESET voltage and (c) normalized resistance for the flat and rough surface condition under the air and vacuum ambience.

with rough surface has higher switching probability. The higher switching probability of $\mathrm{ZnO}$ with rough surface can be attributed to larger interface area, which leads to the more $\mathrm{O}_{2(a d)}^{-}$absorption. It had been shown that higher $\mathrm{O}_{2(a d)}^{-}$ concentration leads to better switching functionality [3]. Furthermore, it can also be observed that the switching probability in air ambience is much better than that under vacuum condition, which can also be attributed to the higher $\mathrm{O}_{2(a d)}^{-}$concentration in air ambience. Additionally, the rough surface condition has stable switching probability against various ambience conditions, which indicates that metal oxide memory with rough surfaces is more suitable to operate as the environment is changed rapidly. The relevant mechanism will be discussed later.

Fig. 3(b) shows the SET/RESET switching voltage of ReRAM with different surface under air and vacuum conditions. For the flat surface condition, the RESET switching voltage measured in the air ambience is lower than that under vacuum condition. It can be attributed to the fact that in the air ambience, the high concentration of $\mathrm{O}_{2(a d)}^{-}$increases the annihilation probability of $\mathrm{V}_{\mathrm{O}}$ 's, and thus the required RESET voltage is reduced as compared with that under vacuum condition. Note that the significant effect of $\mathrm{O}_{2(a d)}^{-}$on annihilation of Vo's and on the formation/rupture of conductive nanofilaments had been previously demonstrated in the literature [3]. Oppositely, the SET process relies on the generation of the $\mathrm{V}_{\mathrm{O}}$ 's. Thus the SET voltage is low under vacuum due to the low concentration of $\mathrm{O}_{2(a d)}^{-}$. As for the ReRAMs with rough surface, the electrical property is stable in various ambiences, compared to that with flat surface condition. Nanoscale rough surfaces would create more surface states with more dangling bonding, implying more complex and stronger bonding to $\mathrm{O}_{2(a d)}^{-}$. Accordingly, the $\mathrm{ZnO}$ ReRAMs with rough surface show relatively invariant SET and RESET voltages.

Fig. 3(c) shows the HRS and LRS resistance for both flat and rough surface conditions. Note that HRS resistance is normalized to its corresponding LRS resistance in the figure for 


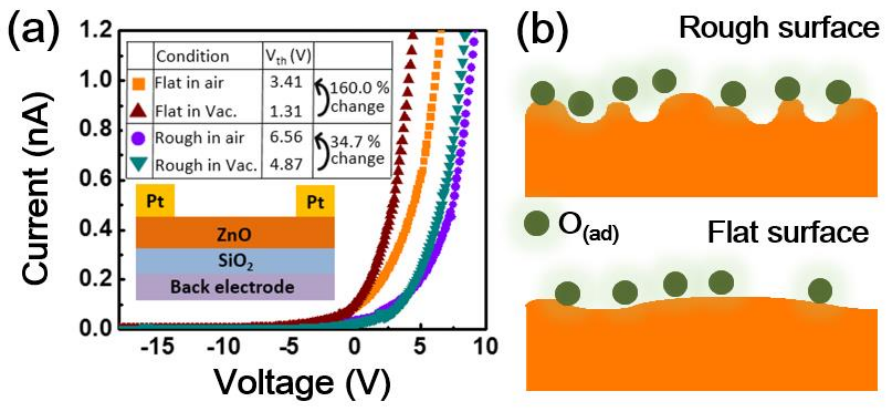

Fig. 4. (a) The transfer characteristics of the ZnO FET with various surface and measuring ambience conditions. The inset in (a) is FET measuring configuration. (b) A schematic of oxygen chemisorption process on the rough and flat surface.

a comparison. It is clear that for the flat surface condition, HRS resistance is ambience-dependent. Indeed, in the air ambience, more $\mathrm{O}_{2(a d)}^{-}$are chemisorbed on the $\mathrm{ZnO}$ surface. The oxygen adsorption leads the band bending of the $\mathrm{ZnO}$ surface, which results in the increase of surface resistance. On the other hand, no ambience-dependent resistance is observed in the LRS. It can be attributed to the fact that the metallic behavior of the conductive nanofilament is not affected by the oxygen chemisorption. Comparing switching voltage and resistance for both surface conditions, the $\mathrm{ZnO}$ ReRAMs with rough surface show relatively invariant HRS values as the ambience is changed, which is consistent to the results of SET and RESET voltages shown in Fig. 3(b).

To further confirm the electrical stability of ZnO ReRAM with rough surface, the electrical characteristics of $\mathrm{ZnO}$ FET configuration (inset of Fig. 4(a)) were studied. Note that, in this study, $V_{\text {th }}$ is the linear extrapolation of $\mathrm{I}_{\mathrm{D}}-\mathrm{V}_{\mathrm{G}}$ curve at its maximum slope. As shown in Fig. 4(a), for both surface conditions, the $\mathrm{V}_{\text {th }}$ of $\mathrm{ZnO}$ under vacuum condition is smaller than that in the air ambience. This is because that the $\mathrm{O}_{2(a d)}^{-}$ concentration on the surface of $\mathrm{ZnO}$ under air condition is greater than that under vacuum condition. The higher $\mathrm{O}_{2(a d)}^{-}$

concentration leads to larger chemisorption-induced band bending which requires higher gate voltage to compensate and hence results in higher $\mathrm{V}_{\text {th }}$ [1]. In addition, the $\mathrm{ZnO}$ with rough surface absorbs more oxygen adatoms due to the larger surface area leading to the larger $\mathrm{V}_{\text {th }}$ than $\mathrm{ZnO}$ with flat surface, as shown in Fig. 4(b). Fig. 4(a) also shows that the difference of $V_{\text {th }}$ between air and vacuum ambience under rough surface condition is less than that under flat surface condition. This indicates that the variation of $\mathrm{O}_{2(a d)}^{-}$concentration absorbed on the rough $\mathrm{ZnO}$ surfaces is much small between air and vacuum ambience, which is consistent with the experimental observation from the memory characterizations (Fig. 3).

\section{CONCLUSION}

In summary, we demonstrated that the surface roughness has significant impact on memory characteristics of ReRAM. The $\mathrm{ZnO}$ ReRAM with rough surfaces shows superior switching probability and stable characteristics against various ambience conditions. The underlying mechanism is ascribed to the roughness-enhanced absorption on oxide surfaces, which leads to the improvement of chemisorption-assisted switching process. These findings not only provide further insights to the surface effect on memory switching behaviors but also help in developing stable and uniform ReRAMs for practical applications.

\section{REFERENCES}

[1] C. Y. Chen, M. W. Chen, J. J. Ke, C. A. Lin, J. R. D. Retamal, and J. H. $\mathrm{He}$, "Surface effects on optical and electrical properties of $\mathrm{ZnO}$ Nanostructures," Pure Appl. Chem., vol. 82, no. 11, pp. 2055-2073, Aug. 2010. doi: 10.1351/PAC-CON-09-12-05.

[2] D. Kang, H. Lim, C. J. Kim, I. Song, J. C. Park, and Y. S. Park, "Amorphous gallium indium zinc oxide thin film transistors: sensitive to oxygen molecules," Appl. Phys. Lett., vol. 90, no. 19, pp. 192101, May 2007. doi: $10.1063 / 1.2723543$.

[3] J. J. Ke, Z. J. Liu, C. F. Kang, S. J. Lin, and J. H. He, "Surface effect on resistive switching behaviors of ZnO," Appl. Phys. Lett., vol. 99, no. 19, pp. 192106, Nov. 2011. doi: 10.1063/1.3659296.

[4] P. K. Yang, W. Y. Chang, P. Y. Teng, S. F. Jeng, S. J. Lin, P. W. Chiu, and J. H. He, "Fully transparent resistive memory employing graphene electrodes for eliminating undesired surface effects," Proc. IEEE., vol. 101, no. 7, pp. 1732-1739, May 2013. doi: 10.1109/JPROC.2013.2260112.

[5] T. H. Huang, P. K. Yang, D. H. Lien, C. F. Kang, M. L. Tsai, Y. L. Chueh, and J. H. He, "Resistive memory for harsh electronics: immunity to surface effect and high corrosion resistance via surface modification," Sci. Rep., vol. 4, pp. 4402, Mar. 2014. doi: 10.1038/srep04402.

[6] A. Dolatshahi-Pirouz, M. B. Hovgaard, K. Rechendorff, J. Chevallier, M. Foss, and F. Besenbacher, "Scaling behavior of the surface roughness of platinum films grown by oblique angle deposition," Phys. Rev. B, vol. 77, no. 11, pp. 115427, Mar. 2008. doi: 10.1103/PhysRevB.77.115427.

[7] H. Inoue, S. Yasuda, H. Akinaga, and H. Takagi, "Nonpolar resistance switching of metal/binary-transition-metal oxides/metal sandwiches: Homogeneous/inhomogeneous transition of current distribution," Phys. Rev. B, vol. 77, no. 3, pp. 035105, Feb. 2008. doi: 10.1103/PhysRevB.77.035105.

[8] T. H. Huang, P. K. Yang, W. Y. Chang, J. F. Chien, C. F. Kang, M. J. Chen, and J. H. He, "Eliminating surface effects via employing nitrogen doping to significantly improve the stability and reliability of $\mathrm{ZnO}$ resistive memory," J. Mater. Chem. C, vol. 1, no. 45, pp. 7593, Sep. 2013. doi: 10.1039/C3TC31542H.

[9] R. Waser, R. Dittmann, G. Staikov, and K. Szot, "Redox-based resistive switching memories-nanoionic mechanisms, prospects, and challenges," Adv. Mater., vol. 21, no. 25, pp. 2632, Jul. 2009. doi: 10.1002/adma.200900375. 